\title{
recildunds
}

Revista Cientifica Mundo de la Investigación y el Conocimiento

\author{
Olga Mariela Herrera Checa a; Andrea Leonor Rodríguez Ramos ${ }^{\text {b; }}$ Luis Alberto \\ Barrera Delgado ${ }^{c}$; María Fernanda Sánchez Pilozo ${ }^{d}$
}

Bocio multinodular tratamiento quirúrgico

Multinodular bocio surgical treatment

Revista Científica Mundo de la Investigación y el Conocimiento. Vol. 3 núm. 4., diciembre, ISSN: 2588-073X, 2019, pp. 150-176

DOI: $10.26820 /$ recimundo/3.(4).diciembre.2019.150-176

URL: http://recimundo.com/index.php/es/article/view/657

Código UNESCO: 3205 Medicina Interna

Tipo de Investigación: Artículo de Revisión

(C) RECIMUNDO; Editorial Saberes del Conocimiento, 2019

Recibido: 15/09/2019

Aceptado: 23/11/2019

Publicado: 30/12/2019

Correspondencia: olgamariela_777@ hotmail.com

a. Médico; Investigador Independiente; Guayaquil, Ecuador; olgamariela_777@ hotmail.com

b. Médico; Investigador Independiente; Guayaquil, Ecuador; aleorre09@ gmail.com

c. Médico; Investigador Independiente; Guayaquil, Ecuador; 1 barrera d@ @otmail.com

d. Médico; Investigador Independiente; Guayaquil, Ecuador; mafercitakris@ gmail.com 


\section{Bocio multinodular tratamiento quirúrgico}

Vol. 3, núm. 4., (2019)

Olga Mariela Herrera Checa; Andrea Leonor Rodríguez Ramos; Luis Alberto Barrera Delgado;

María Fernanda Sánchez Pilozo

\section{RESUMEN}

La existencia de un crecimiento rápido de la glándula tiroides, aunado a la aparición de síntomas compresivos, hipertiroidismo o la sospecha de malignidad, son indicaciones que están bien establecidas para esta enfermedad. En estos supuestos se debe realizar una tiroidectomía. Al encontrarse con el bocio multinodular originado por el aumento irregular de tamaño de la glándula tiroides que supone una elevación de la TSH. Esta hiperplasia tiroidea se debe probablemente a una disminución de la producción de hormonas tiroideas con relación a las necesidades del organismo. Su parición puede ser de origen congénito o adquirido de crecimiento asimétrico. En un nódulo predominante, es imprescindible realizar una punción con aguja fina para poder establecer con seguridad la extensión de la tiroidectomía. Existen diferentes modalidades quirúrgicas en el tratamiento del bocio multinodular que varían en función de su extensión y preferencias de cada cirujano; pues, la enfermedad muestra una tendencia a producir un cambio multinodular en toda la glándula, aunque el bocio puede aparecer inicialmente como un nódulo solitario en un lóbulo o área de la glándula, con el tiempo puede generalizarse. El procedimiento quirúrgico puede ser unilateral o bilateral y la resección varía desde una simple enucleación a una tiroidectomía total (TT). Algunos cirujanos defienden una cirugía radical que evite recidivas. Otros especialistas son menos agresivos y practican resecciones menores, por tener menor morbilidad postoperatoria y evitar una dependencia hormonal farmacológica de por vida. Consideraciones relevantes para la realización del presente artículo, cuyo objetivo es describir el bocio multinodular tratamiento quirúrgico, conducido bajo una investigación documental bibliográfica, lo que permite concluir en relación al tema seleccionado que la realización de este tratamiento quirúrgico depende de las características presente en el bocio multinodular y es allí, donde la selección del procedimiento es debidamente implementado por el cirujano de acuerdo con su criterio.

Palabras Claves: Tiroides; Bocio multinodular; Tratamiento quirúrgico. 


\section{Bocio multinodular tratamiento quirúrgico}

Vol. 3, núm. 4., (2019)

Olga Mariela Herrera Checa; Andrea Leonor Rodríguez Ramos; Luis Alberto Barrera Delgado; María Fernanda Sánchez Pilozo

\section{ABSTRACT}

The existence of rapid growth of the thyroid gland, in addition to the onset of compressive symptoms, hyperthyroidism or suspected malignancy, are indications that are well established for this disease. In these cases, a thyroidectomy should be performed. When faced with the multinodular goiter caused by the irregular increase in the size of the thyroid gland that implies an elevation of TSH. This thyroid hyperplasia is likely due to a decrease in thyroid hormone production relative to the body's needs. Its partition may be of congenital origin or acquired of asymmetrical growth. In a predominant nodule, it is essential to perform a fine needle puncture in order to safely establish the extent of the thyroidectomy. There are different surgical modalities in the treatment of multinodular goiter that vary depending on its extent and preferences of each surgeon; therefore, the disease shows a tendency to produce a multinodular change throughout the gland, although the goiter may initially appear as a solitary nodule in a lobe or area of the gland, over time it may become widespread. The surgical procedure can be unilateral or bilateral and resection varies from a simple enucleation to a total thyroidectomy (TT). Some surgeons advocate radical surgery that prevents recurrence. Other specialists are less aggressive and practice minor resections, for having less postoperative morbidity and avoiding a lifelong pharmacological hormonal dependence. Considerations relevant to the realization of this article, the objective of which is to describe the multinodular goiter surgical treatment, conducted under a bibliographic documentary research, which allows to conclude in relation to the selected subject the realization of this surgical treatment depends on the characteristics present in the multinodular goiter and it is there, where the selection of the procedure is duly implemented by the surgeon according to his criteria.

Keywords: Thyroid; goth-gononodular; surgical treatment. 


\section{Bocio multinodular tratamiento quirúrgico}

Vol. 3, núm. 4., (2019)

Olga Mariela Herrera Checa; Andrea Leonor Rodríguez Ramos; Luis Alberto Barrera Delgado;

María Fernanda Sánchez Pilozo

\section{Introducción.}

La tiroides es una glándula en forma de mariposa ubicada en el cuello, justo arriba de la clavícula, encargada de producir hormonas tiroideas que controlan el ritmo de muchas actividades del cuerpo. Estas influyen con la velocidad con la que se queman calorías y cuán rápido late el corazón. Todas estas actividades componen el metabolismo del cuerpo. Aunque su peso está en torno a los 30 gramos, tiene una influencia destacada en el estado de salud. Es una glándula endocrina que a través de la secreción de sus hormonas participa en casi todas las funciones básicas del organismo, como, por ejemplo: Regula el metabolismo y temperatura corporal, es necesaria para el crecimiento, el sistema nervioso necesita de ella para su correcto desarrollo, regula la asimilación de los diferentes nutrientes, es fundamental para regular el ritmo cardiaco y el desarrollo de la piel. (Copello Millares, Merino Rodríguez, \& Carralero Zaldívar, 2016)

La tiroides necesita yodo para segregar sus hormonas que equilibran el metabolismo (velocidad a la que se consume la energía proveniente de los alimentos), y ayudan a quemar el exceso de grasa regulando eficazmente el nivel energético. Desempeña un amplio abanico de funciones, en el control del peso, nivel de colesterol en la sangre, mantenimiento de la fortaleza muscular y estado de la piel. Una tiroides sana es básica para gozar de un bienestar general. El trastorno más común de la tiroides se produce por una baja actividad de dicha glándula. Este trastorno es conocido como hipotiroidismo. La misma tiene forma de pajarita. Sus dos lóbulos se sitúan a ambos lados de la tráquea en la parte frontal de la garganta y se unen por una tira de tejido denominada istmo. Aunque se encuentra cercana a la piel, la tiroides es normalmente pequeña y blanda, apenas puede ser palpada a través del contacto con la piel. Además, está 


\section{Bocio multinodular tratamiento quirúrgico}

Vol. 3, núm. 4., (2019)

Olga Mariela Herrera Checa; Andrea Leonor Rodríguez Ramos; Luis Alberto Barrera Delgado; María Fernanda Sánchez Pilozo

compuesta por dos conjuntos de células que segregan hormonas cuya producción depende de la capacidad del organismo de extraer el suficiente yodo de los alimentos ingeridos. (Martin, 2016)

Para el diagnóstico de enfermedades de la tiroides, los médicos usan su historia clínica, un examen físico y pruebas para la tiroides. A veces también utilizan una biopsia. El tratamiento depende del problema, pero puede incluir medicamentos, terapia con yodo radiactivo o cirugía de tiroides. Generalmente las actividades relacionadas con la evaluación del paciente ante problemas tiroideos, se encuentran determinados por la presencia de diferentes síntomas entre los cuales se encuentran: cansancio, fatiga, taquicardia, aumento del apetito, sudoración, pérdida y aumento de peso entre otros, la deficiencia de yodo es la causa más común del bocio. El cuerpo necesita yodo para producir la hormona tiroidea. Si no obtiene de manera suficiente en su dieta, la tiroides se agranda para tratar de capturar todo el yodo que pueda y producir la cantidad adecuada de hormona tiroidea

Sin embargo, cuando la consulta médica diagnostica en el paciente: intolerancia al calor, aumento de la transpiración, calambres musculares, periodos menstruales irregulares y nerviosismo, además, se acompaña con los exámenes de laboratorios y eco tiroideo, que hace posible observar un aumento de manera irregular por culpa de un estímulo anormal de una hormona hipofisaria (TSH) formando múltiples nódulos produciendo habitualmente un aumento del diámetro del cuello, esto confirma la presencia del bocio multinodular.

En este sentido, Vidal (2017) define al bocio multinodular "como una alteración en toda la glándula que crece de manera irregular por culpa de un estímulo anormal de una hormona hipofisaria (TSH) formando múltiples nódulos produce habitualmente un aumento 


\section{Bocio multinodular tratamiento quirúrgico}

Vol. 3, núm. 4., (2019)

Olga Mariela Herrera Checa; Andrea Leonor Rodríguez Ramos; Luis Alberto Barrera Delgado; María Fernanda Sánchez Pilozo

del diámetro del cuello.” (p.10). Además, destaca que es una enfermedad que predomina más en mujeres y tiene una incidencia variable dentro de un mismo territorio, para su tratamiento se precisa como única solución definitiva la cirugía.

Es importante acotar, que una vez diagnosticada la presencia del bocio multinodular, el especialista debe recurrir a la aplicación del respectivo tratamiento, en este caso particular, corresponde a lo quirúrgico, todo depende de la extensión de la enfermedad y preferencias del cirujano. La enfermedad puede aparecer inicialmente como un nódulo solitario, pero con el tiempo puede llegar a generalizarse. Aunque la edad no es contraindicación para la cirugía tiroidea, pocos pacientes añosos son intervenidos, pues, existe un mayor riesgo de morbilidad. (Rodríguez Ramos, Boffill Corrales, \& Rodríguez Soria, 2016)

Por ello, para llevar a cabo este tratamiento quirúrgico el cirujano especilista debe considerar la existencia de un crecimiento rápido de la glándula tiroides, aparición de síntomas comprensivos, hipertiroidismo, o sospecha de malignidad, son indicaciones que estiman la realización de una tiroidectomía. En los casos de bocio multinodular de crecimiento asimétrico o los que presenten un nódulo predominante, es imprescindible realizar una punción con aguja fina para poder establecer con seguridad la extensión del respectivo proceso quirúrgico.

Según lo anterior, se puede entender que la indicación de cirugía en los bocios multinodulares pequeños, clínicamente asintomáticos y sin ningún nódulo predominante, es controvertida. La tiroidectomía en fases asintomáticas puede ser considerada como cirugía profiláctica y podría estar indicada en pacientes jóvenes con historia familiar de bocio 


\section{Bocio multinodular tratamiento quirúrgico}

Vol. 3, núm. 4., (2019)

Olga Mariela Herrera Checa; Andrea Leonor Rodríguez Ramos; Luis Alberto Barrera Delgado; María Fernanda Sánchez Pilozo

multinodular sintomático. En relación al bocio multinodular en pacientes de edad avanzada, se efectúa en el caso de presentar síntomas compresivos o alteraciones analíticas, debe plantearse la resección quirúrgica como una alternativa. Mientras que los casos en que es descubierto de manera casual en el contexto del estudio de otra enfermedad, es importante individualizar el tratamiento del bocio multinodular teniendo en cuenta el contexto del paciente. (Sosa Martín \& Ernand Rizo, 2016)

Los planteamientos citados, permiten destacarlos como informaciones significativas para el desarrollo del artículo que se presenta de forma descriptiva para lograr una mejor apreciación del contexto relativo al tema previamente seleccionado con el fin de generar nuevas consideraciones generales, pertinentes a las interpretaciones vinculadas con los aportes encargados de ampliar cada uno de los elementos que estructuran el hacer científico.

\section{Método.}

Los esquemas investigativos, para logra eficiencia en las actividades previamente planificadas, de concebir el acto de indagar desde una perspectiva metodológica que caracterice cada momento considerado como evento esencial que dará apertura a la construcción de constructos viables y proclives a lograr apreciaciones generales como resultado a un manejo innovador de los medios documentales.

Por ello, el presente proceso de investigación visualiza la introducción del método como una herramienta viable que propicia el análisis exhaustivo de los acontecimientos debidamente relacionados entre sí para lograr la redimensión de nuevos conceptos que dan cabida a la presencia de explicaciones ajustada al criterio dado por, Pernalete (2016) el método en el campo 


\section{Bocio multinodular tratamiento quirúrgico}

Vol. 3, núm. 4., (2019)

Olga Mariela Herrera Checa; Andrea Leonor Rodríguez Ramos; Luis Alberto Barrera Delgado; María Fernanda Sánchez Pilozo

científico "es un medio operativo que hace posible la recopilación de informaciones de forma directa e indirecta para luego facilitar una apreciación global de los hechos en estudio".(p.36). En consecuencia, su introducción en el desarrollo de este artículo asegura primeramente la revisión coherente de la información para luego darle su respectiva organización definitiva.

En esa misma dirección se puede indicar, que para dar las respectivas acciones de indagación y valoración de las técnicas, fue necesario incluir un método de apoyo que sustente el trabajo del investigador, momento que condujo a la aplicación del método, inductivo, que desde la perspectiva de Peña (2016), consiste en la organización de materiales que tienen interés y que facilitan el planteamiento de ideas particulares a fin de expresar generalidades" (p. 92). Es decir, los aportes surgen de interpretaciones que son llevadas a cabo de forma individual, para luego compaginarlas y construir el contexto general del tema a tratar.

\section{Tipo de Investigación}

En función al uso de un método estimado por la revisión de aportes referenciales bibliográficos, lleva a considerar que la investigación se encuentra debidamente conectada con el tipo documental monográfico, al respecto, (Pernalete, 2016) como: "es el estudio de problemas con el propósito de ampliar y profundizar el conocimiento de su naturaleza, con apoyo principalmente, en trabajos previos, información y datos divulgados por medios impresos, audiovisuales o electrónicos". (p.13). Por lo tanto, se procedió a la revisión exhaustiva de aquellos documentos, libros, revistas, estudios previos, entre otros estrechamente vinculados con la temática a indagar. 


\section{Bocio multinodular tratamiento quirúrgico}

Vol. 3, núm. 4., (2019)

Olga Mariela Herrera Checa; Andrea Leonor Rodríguez Ramos; Luis Alberto Barrera Delgado; María Fernanda Sánchez Pilozo

De este modo, se cumplieron una serie de acciones vinculadas con la revisión de diversos materiales bibliográficos que de una u otra forma se encargan de dar la respectiva consistencia a la temática a tratar. Asimismo, se puede decir que se ubica en el tipo teórico-reflexivo y analístico, según Pernalete (ob.cit), “actúan sobre situaciones prácticas y problemas en el área de la especialidad, con el fin de describirlos, identificar factores intervinientes o posibles causas y vías para su solución”. (p.16). Es decir, mediante este proceso se logró entender, sistematizar y valorar las condiciones que determinan las acciones propuestas en cuanto a la descripción del bocio multimodular tratamiento quirúrgico, con miras a generar un cuerpo de apreciaciones reflexivas que puedan ayudar a entender la necesidad de gestar cambios a nivel de los diversos especialistas encargados de lograr la respectiva valoración del paciente con enfermedades tiroideas y ofrecer de este modo seguridad social y personal

Asimismo, el autor citado, destaca que los estudios documentales monográficos, son conceptualizados como: "trabajos orientados al abordaje de la información, organización, análisis crítico, reflexivo, interpretación y síntesis de referencias y otros insumos pertinente al tema seleccionado". (p.15). Por ello, se, pudo dar a cada interpretación su respectiva ubicación en un orden teórico, con el fin de ajustar al propósito preestablecidos para su realización de forma valorativa.

\section{Fuentes Documentales}

Para garantizar el desarrollo de cada fase implícita en el proceso investigativo, se hizo necesario recurrir a la implementación de fuentes caracterizadas por estimar la lectura previa con un valor esencial para encontrar los diferentes documentos y cumplir con su revisión e 


\section{Bocio multinodular tratamiento quirúrgico}

Vol. 3, núm. 4., (2019)

Olga Mariela Herrera Checa; Andrea Leonor Rodríguez Ramos; Luis Alberto Barrera Delgado; María Fernanda Sánchez Pilozo

interpretación, dando así la respectiva flexibilidad metodológica en perfecta correspondencia con el método previamente seleccionado. Tal como lo expresa, Peña (ob.cit) "son medios que sirven de apoyo para cumplir con la realización de valoraciones conjunta referidas a un tema en particular". (p.24). En otras palabras, su incorporación hizo posible desglosar los contenidos a tratar de forma coherente y funcional para finalmente obtener informaciones viables encargadas de asegurar el desarrollo investigativo.

\section{Técnicas para la Recolección de la Información}

En las investigaciones de tipo documental bajo la dirección del diseño bibliográfico, el uso de las técnicas va a estar determinado por la presencia de eventos que guardan directamente una correspondencia con las características del proceso de indagación. Por lo tanto, en este estudio se asumieron con técnicas las documentales donde se emplearon: el fichaje y resumen, la primera con la finalidad de seleccionar los diversos autores, títulos de trabajos, fuentes primarias de las informaciones relacionadas con el tema, para luego adecuarlo a la implementación del resumen como medio que permitió asegurar la escogencia de los tópicos requeridos en el trabajo, tal como lo afirma, Peña (ob.cit), las técnicas documentales centradas en el fichaje y resumen “consisten en la relación existente entre el autor y el documento escrito en función a un tiempo, lugar y circunstancia" (p. 27).

\section{Resultados.}

El desarrollo de los aspectos que se insertan a continuación, guardan relación con los constructos encargados de estructurar el proceso investigativo y convertirlo en un aporte científico de interés dentro del campo de las ciencias médicas, mediante su presentación se logra 


\section{Bocio multinodular tratamiento quirúrgico}

Vol. 3, núm. 4., (2019)

Olga Mariela Herrera Checa; Andrea Leonor Rodríguez Ramos; Luis Alberto Barrera Delgado; María Fernanda Sánchez Pilozo

visualizar y comprender la actuación del cirujano ante la presencia de enfermedades de tipo tiroideas.

\section{Glándula Tiroides}

La tiroides trabaja conjuntamente con otras dos glándulas productoras de hormonas, la pitituaria y el hipotálamo. Esta acción tripartita tiene como objetivo la producción de la cantidad justa de energía que el organismo necesita en cada momento (baja durante el sueño, alta durante la vigilia, más alta aún durante la actividad física). Millones de células foliculares segregan hacia la sangre las hormonas que contienen yodo. La más importante de dichas hormonas es la tiroxina (T4), que representa el $99.9 \%$ de las hormonas producidas por la tiroides. Otra hormona, la triyodotironina (T3), supone el $0.1 \%$ restante. Las células restantes, conocidas como parafoliculares, se hallan aisladas o en pequeños grupos y segregan una hormona denominada calcitonina. 


\section{Bocio multinodular tratamiento quirúrgico}

Vol. 3, núm. 4., (2019)

Olga Mariela Herrera Checa; Andrea Leonor Rodríguez Ramos; Luis Alberto Barrera Delgado; María Fernanda Sánchez Pilozo

\section{Imagen $\mathbf{N}^{\circ} \mathbf{1}$ Glándula Tiroides}

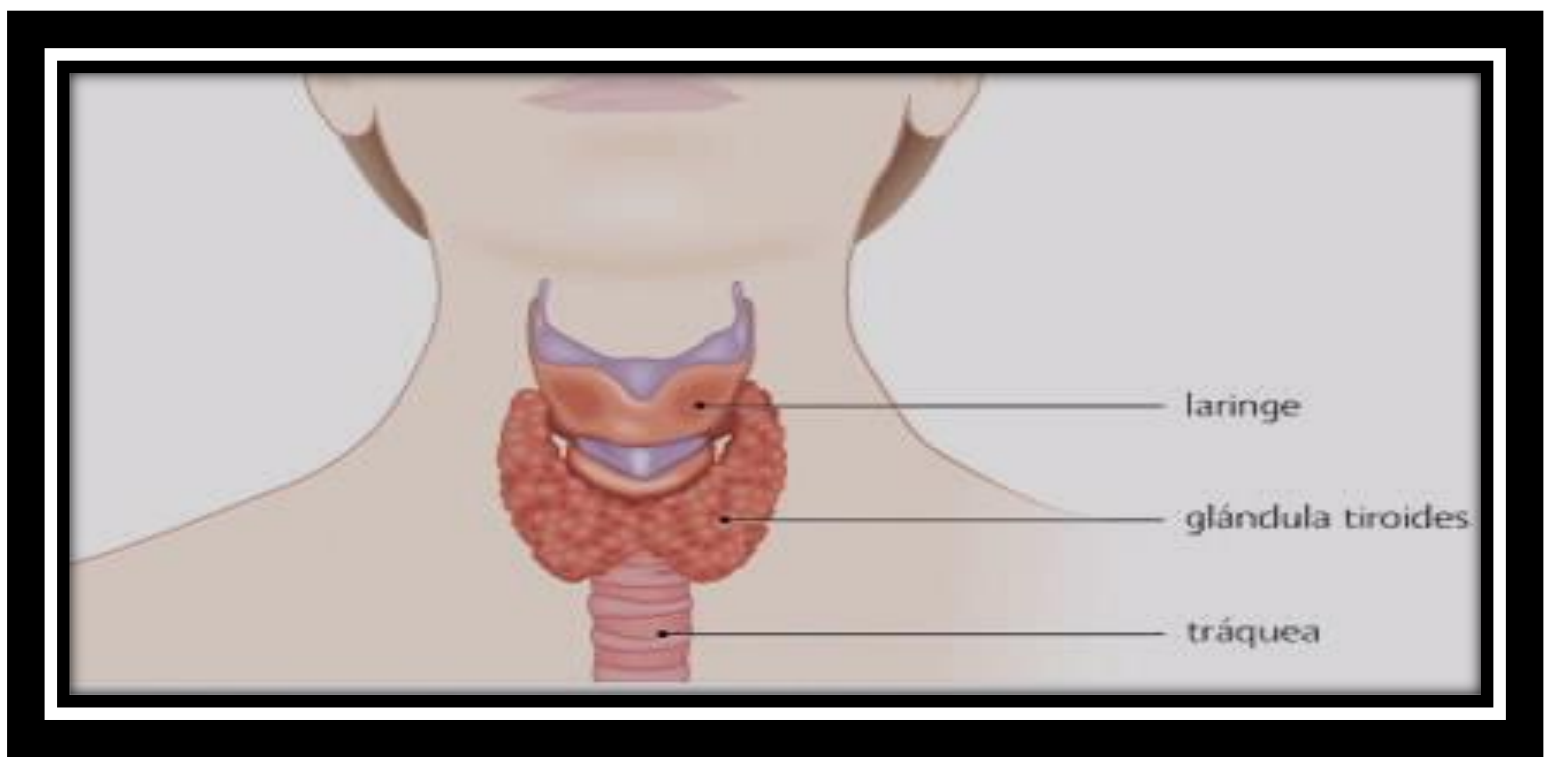

Fuente: Vidal (ob.cit)

La pituitaria, una glándula del tamaño de un guisante situada en la base del cerebro, controla el nivel de hormonas segregadas por la tiroides y a su vez está controlada por el hipotálamo. Las hormonas T3 y T4, segregadas por la tiroides, son fundamentales para el control y mantenimiento de las funciones vitales del organismo. A través de su papel en la regulación del metabolismo y en el equilibrio del calcio en el organismo, dichas hormonas son básicas para el crecimiento físico y el desarrollo mental de los niños. El calcio es clave para mantener unos huesos saludables, y su nivel en el organismo se regula por la calcitonina, las hormonas tiroideas y la paratiroides. Dicha hormona, la calcitonina, es producida por cuatro glándulas paratiroideas localizadas justo detrás de la tiroides. 


\section{Bocio multinodular tratamiento quirúrgico}

Vol. 3, núm. 4., (2019)

Olga Mariela Herrera Checa; Andrea Leonor Rodríguez Ramos; Luis Alberto Barrera Delgado; María Fernanda Sánchez Pilozo

El trastorno más común de la tiroides se produce por una baja actividad de dicha glándula conocido como hipotiroidismo, cunado la tiroides no llega a producir las hormonas necesarias debido a una insuficiente ingesta de yodo. Es una enfermedad que puede pasar desapercibida durante muchos años, al poder dar síntomas inespecíficos y desarrollarse lentamente, por lo cual el enfermo y sus familiares no advierten esos cambios hasta que la enfermedad está muy avanzada. Por ello, el hipotiroidismo puede permanecer años sin ser diagnosticado, pues, los síntomas, tales como el olvido y fatiga, pueden ser fácilmente confundidos con otros estados físicos como el envejecimiento natural, menopausia o estrés. Entre otros síntomas se pueden citar ronquera, languidez, apatía, dificultad al tragar, intolerancia al frío, ganancia de peso, edemas en las piernas, somnolencia, aspereza en la piel.

Sin embargo, el hipotiroidismo moderado puede ser detectado por un sencillo análisis de sangre y se trata fácilmente mediante hormonas sintéticas en pastillas, a modo de suplemento o sustitutivo de las hormonas producidas de manera natural por el organismo. Dicha medicación es diaria y para toda la vida. Por ello, es importante mantener un índice de sospecha elevado, y ante pacientes (sobre todo mujeres), que presenten estos síntomas inespecíficos pero persistentes, realizar la analítica mencionada.

Por otro lado, en la enfermedad conocida como tiroiditis crónica (que también puede producir hipotiroidismo), la tiroides puede agrandarse, algunas veces de forma considerable, dando lugar a una inflamación conocida como bocio. Dicha inflamación tiene lugar cuando el sistema inmunológico produce anticuerpos que atacan a la tiroides, estimulando a la pituitaria para segregar un mayor número de hormonas, lo que hace que la tiroides tenga más trabajo y aumente de tamaño. 


\section{Bocio multinodular tratamiento quirúrgico}

Vol. 3, núm. 4., (2019)

Olga Mariela Herrera Checa; Andrea Leonor Rodríguez Ramos; Luis Alberto Barrera Delgado; María Fernanda Sánchez Pilozo

En muchos países, el bocio ha sido ampliamente eliminado por la difusión del uso de sal yodada. Asimismo, el hipotiroidismo puede ser resultado de una disfunción glandular conocida como mixedema en los adultos y cretinismo (hipotiroidismo congénito) en los niños. El hipertiroidismo, es menos frecuente que el hipotiroidismo, se produce cuando la glándula tiroides segrega demasiadas hormonas. También es conocido como enfermedad de Graves o Bocio tóxico difuso. Es más común en las mujeres, particularmente en aquellas que se encuentran entre los 30 y los 40 años de edad.

Además del bocio, los síntomas incluyen trastornos del sueño (insomnio), debilidad muscular, temblores, palpitaciones, irregularidad en la menstruación, intolerancia al calor, pérdida de peso a pesar de tener buen apetito o problemas de visión. El tratamiento para reducir la producción de hormonas con medicamentos puede ser complejo y largo en el tiempo. También puede usarse la cirugía para combatir el hipertiroidismo. Existen otros trastornos de la tiroides pero no son muy frecuentes. Entre ellos enfermedades congénitas, infecciones, quistes, y tumores benignos o malignos.

El bocio multinodular es otra causa común de bocio. Individuos con este problema tienen uno o varios nódulos dentro de la glándula lo cual resulta en una tiroides agrandada. Muchas veces esto se detecta como una sensación nodular cuando se palpa la glándula tiroides en el examen físico. Los pacientes pueden presentarse con un nódulo grande único o con muchos nódulos pequeños en la glándula. De tal manera que en etapas tempranas del bocio multinodular, cuando hay muchos nódulos pequeños el tamaño de la glándula puede aún no estar aumentado. A diferencia de otros bocios que hemos discutido, no se entiende por completo la causa de este tipo de bocio. Además de las causas comunes de bocio mencionadas anteriormente, hay otras causas 


\section{Bocio multinodular tratamiento quirúrgico}

Vol. 3, núm. 4., (2019)

Olga Mariela Herrera Checa; Andrea Leonor Rodríguez Ramos; Luis Alberto Barrera Delgado; María Fernanda Sánchez Pilozo

menos comunes. Algunas de ellas son debidas a defectos genéticos, otras a lesiones e infecciones de la glándula tiroides o secundaria a tumores (cancerígenos y benignos).

Las hormonas tiroideas son reguladores de múltiples factores cuestiones como: temperatura corporal, peso, estado de ánimo o frecuencia cardiaca. Así, existen múltiples patologías relacionadas con las tiroides como: el hipotiroidismo cuando la glándula tiroides produce más hormonas de lo que el cuerpo necesita e hipertiroidismo cuando la tiroides no produce suficientes hormonas; así como el bocio agrandamiento de la glándula tiroides, el cual puede ser difuso, nodular y multinodular; y el cáncer de tiroides. El número de casos de enfermedad tiroidea, tanto la diagnosticada como la no diagnosticada se estima en un $9 \%$ de la población española, que llega hasta el 13,5\% en mayores de 60 años, con especial incidencia en mujeres.

El cáncer de tiroides supone el 3,8\% del total mundial de nuevos casos de cáncer, aunque sólo es responsable del $0,3 \%$ de la mortalidad por cáncer sólo 1 de cada 1.000 personas que fallecen por cáncer lo hacen por el de tiroides. De hecho, el índice de supervivencia a los cinco años de ser detectada la enfermedad es del 97\%. A nivel de bocios y nódulos tiroideos se tratan más de 3.000 casos nuevos por año, mientras que hay unos 50 cánceres de tiroides detectados cada año, y se hace un seguimiento de entre 4000 y 5000 casos de cáncer. En Ecuador el 65 por ciento de los casos de hipotiroidismo en el país son provocados por reacciones autoinmunes hacia la glándula de la tiroides, el 22 por ciento por déficit de yodo y el 1 por ciento por fármacos que bloquean la producción de hormonas tiroideas. Asimismo, Sánchez (2016) precisa que en Ecuador los estudios epidemiológicos estiman que la prevalencia de las alteraciones de la 


\section{Bocio multinodular tratamiento quirúrgico}

Vol. 3, núm. 4., (2019)

Olga Mariela Herrera Checa; Andrea Leonor Rodríguez Ramos; Luis Alberto Barrera Delgado; María Fernanda Sánchez Pilozo

función tiroidea en adultos es del 1 a al 4\%, siendo superior en mujeres y personas con síndrome de Down y aumentando con la edad.

De igual manera indica que, se estima una incidencia anual en adultos de 0,05 al $0,1 \%$ para el hipertiroidismo, y del 0,08 al 0,2\% para el hipotiroidismo, siendo mayor en mujeres de edad avanzada. De acuerdo a la alteración con respecto al tamaño tiroideo se estima que aproximadamente el 10\% de la población adulta tiene nódulos tiroideos que son clínicamente importantes por varias razones, pueden causar desde disfunción tiroidea e incluso llegar al cáncer de ésta glándula; teniendo como prevalencia de malignidad desde $4 \%$ a $6.5 \%$ y es totalmente independiente del tamaño del nódulo; éstos son 8 veces más frecuentes en mujeres que en hombres y su prevalencia aumenta con la edad. Sin embargo, esta patología es dos veces más probablemente maligna en hombres que en mujeres. Aunque la mayoría de los nódulos tiroideos son benignos, el 5\% de ellos pueden contener un tumor maligno con una incidencia de alrededor de 25.000 nuevos pacientes con cáncer de tiroides por año, produciendo más de 1.400 muertes anuales.

\section{Bocio Multimodular}

El bocio multinodular se refiere al agrandamiento generalizado de la glándula tiroides con nódulos reconocibles dentro de la misma. La glándula tiroides consiste en dos lóbulos conectados. Los pacientes afectados por bocio a menudo se presentan a la consulta con un agrandamiento no simétrico de la glándula tiroides y un edema visible en el aspecto anterior del cuello. Pueden reconocerse uno o más nódulos. La causa más frecuente del bocio multinodular es la deficiencia de yodo. El bocio no tóxico significa que los nódulos no secretan hormonas 


\section{Bocio multinodular tratamiento quirúrgico}

Vol. 3, núm. 4., (2019)

Olga Mariela Herrera Checa; Andrea Leonor Rodríguez Ramos; Luis Alberto Barrera Delgado; María Fernanda Sánchez Pilozo

tiroideas de forma incontrolada. La tiroidectomía total es una cirugía que incluye la extracción quirúrgica de la glándula tiroides completa. La tiroidectomía casi total es una cirugía que incluye la extracción quirúrgica de ambos lóbulos tiroideos, excepto una cantidad pequeña de tejido tiroideo (en uno o ambos lados menos de 1,0 ml). La tiroidectomía subtotal deja 3 a 5 g en el lado menos afectado de la glándula tiroides.

Cabe destacar que, dicha enfermedad es definido como el aumento del tamaño del tiroides, es la afección más frecuente de la glándula, tanto en su forma difusa como uni o multinodular. Prácticamente, toda la patología tiroidea puede cursar con bocio y la forma de presentación más frecuente de las neoplasias del tiroides, tanto benigna como maligna, es como un nódulo tiroideo, generalmente único. Todo ello hace que la consulta por bocio sea muy frecuente en la práctica clínica endocrinológica y confiere una importancia especial al problema del nódulo tiroideo, que siempre plantea la duda diagnóstica en cuanto a su posible malignidad.

Por ello, es conocida como el aumento de tamaño del tiroides, independientemente de su etiología. El bocio puede clasificarse en difuso o nodular (único o multinodular) y se conoce como bocio simple al que no se debe a la existencia de una enfermedad autoinmunitaria, una tiroiditis o una neoplasia, aunque, en ocasiones, alguno de estos procesos puede acompañarlo. El bocio simple es la enfermedad más común del tiroides y su historia natural demuestra la evolución del aumento de tamaño difuso inicial a la formación de nódulos tiroideos (NT). La prevalencia del trastorno en zonas no endémicas es del 4-7\%, con un predominio en la mujer de 7-13:1, y resulta mayor, en las zonas con endemia, dependiendo de la gravedad del déficit de aporte de yodo. También debe recordarse que tanto el peso como la nodularidad del tiroides aumentan con la edad. 


\section{Bocio multinodular tratamiento quirúrgico}

Vol. 3, núm. 4., (2019)

Olga Mariela Herrera Checa; Andrea Leonor Rodríguez Ramos; Luis Alberto Barrera Delgado; María Fernanda Sánchez Pilozo

El bocio afecta a un 12,8 \% de la población gallega, con mayor incidencia entre mujeres $(11,3 \%)$ que entre hombres $(6,5 \%)$, mientras que en algunas zonas montañosas de Galicia y de interior se detecta un 30\% de población con un déficit de yodo, con especial incidencia entre niños, ancianos y mujeres embarazadas. De este modo, es la primera manifestación de un paciente que puede acabar teniendo un cáncer de tiroides, de tal forma que entre un $4 \%$ y $7 \%$ de las personas con bocio van a tener un carcinoma tiroideo.

Los factores etiológicos del bocio son múltiples y, en ocasiones, pueden asociarse dos o más de ellos. Las causas principales de bocio difuso y de enfermedad nodular tiroidea (ENT) El déficit de aporte yódico es la causa más importante del bocio simple y constituye la etiología fundamental del bocio endémico, que se define cuando más del 10\% de la población general de una zona geográfica presenta bocio. Desde la introducción de los programas de profilaxis yódica, la prevalencia del bocio endémico ha disminuido mucho. La ingesta de yodo que se recomienda para un sujeto adulto oscila entre 150 y 300 g/día.

Otro factor bociógeno importante es el aumento del aclaramiento de yodo por el riñón que puede observarse en la pubertad, el embarazo, la lactancia y el climaterio, especialmente cuando estas situaciones se asocian con cierta insuficiencia en la ingesta de yodo. El exceso de aporte yódico también puede originar la formación de bocio, aunque esta etiología es infrecuente. La forma de presentación clínica más habitual de las neoplasias tiroideas tanto benigna como maligna es la aparición de un NT que ocurre hasta en el 75-90\% de los casos de carcinoma, aunque sólo el 5-15\% de los NT únicos sin antecedentes de irradiación y 20-25\% de los que han sido irradiados son malignos. 


\section{Bocio multinodular tratamiento quirúrgico}

Vol. 3, núm. 4., (2019)

Olga Mariela Herrera Checa; Andrea Leonor Rodríguez Ramos; Luis Alberto Barrera Delgado; María Fernanda Sánchez Pilozo

Por otra parte, la ENT es un motivo de consulta muy frecuente en la práctica clínica endocrinológica su palpación cervical sistemática permite la detección de entre un 4 y un $7 \%$ en la población general, y entre un 20 y un 30\% en la población irradiada. La prevalencia de la ENT es mayor en el sexo femenino, y aumenta con la edad; en estudios necrópsicos, es de más del $50 \%$ y, en exploraciones ecográficas, su hallazgo casual (incidentalomas tiroideos) llega al 67\% (intervalo: 13-67). En cualquier caso, el carcinoma tiroideo, a pesar de ser la neoplasia endocrinológica más común, es poco frecuente (se diagnostican de 36 a 60 nuevos casos clínicamente relevantes por 100.000 habitantes/año).

Los carcinomas llamados ocultos (generalmente papilares), de menos de 1 o $1,5 \mathrm{~cm}$, llegan a ser un hallazgo casual entre el 3 y el 35\% de las necropsias y el 4-17\% de los bocios extirpados por otra causa. El pronóstico del bocio simple es bueno y su evolución desfavorable depende sólo de la posible aparición de fenómenos compresivos. En los estadios iniciales, el proceso puede revertir espontáneamente, especialmente en el varón y después de la pubertad pero, una vez en fase de nodularidad, las alteraciones de la glándula son completamente irreversibles. La evolución del bocio simple hacia el hipotiroidismo es excepcional mientras que el desarrollo de hipertiroidismo es relativamente frecuente (bocio multinodular tóxico).

El tratamiento con levotiroxina sódica puede disminuir de forma transitoria el tamaño del 25\% de los bocios simples siempre que se administre en los primeros meses de evolución y a dosis inhibidoras de la TSH. En la amplia mayoría de casos de bocio el diagnóstico se lleva a cabo por inspección y palpación del médico. La realización de una ecografía tiroidea aporta habitualmente datos adicionales relacionados con la función y la morfología, especialmente en lo 


\section{Bocio multinodular tratamiento quirúrgico}

Vol. 3, núm. 4., (2019)

Olga Mariela Herrera Checa; Andrea Leonor Rodríguez Ramos; Luis Alberto Barrera Delgado; María Fernanda Sánchez Pilozo

que respecta a la existencia de nódulos. La gammagrafía tiroidea puede identificar los nódulos captantes o calientes y los no captantes o fríos.

Para completar el diagnóstico, es necesario realizar una estimulación de hormonas tiroideas y TSH en sangre para saber si la situación es normo, hipo o hiperfuncionante. Cuando se observan formaciones nodulares, puede ser necesario realizar una punción-aspiración con aguja fina para conocer la estructura celular del nódulo y aproximarse hacia su posible benignidad o malignidad. Aparte de los síntomas compresivos, las manifestaciones que puede originar tienen relación con las posibles alteraciones acompañantes de la función tiroidea, como es el hipertiroidismo (nerviosismo, palpitaciones, cansancio, pérdida de peso, entre otras) o el hipotiroidismo (intolerancia al frío cansancio, somnolencia, sequedad de piel. Cuando se investiga mediante ecografía, la frecuencia de bocio aumenta con la edad hasta llegar a un $60 \%$ de casos en mujeres de edad superior a 60 años. No obstante, también puede darse en varones, aunque con menor frecuencia. No es infrecuente que se observe de forma transitoria en recién nacidos de madres tratadas durante el embarazo con fármacos antitiroideos por hipertiroidismo. Es frecuente también su aparición en el curso del embarazo. 


\section{Bocio multinodular tratamiento quirúrgico}

Vol. 3, núm. 4., (2019)

Olga Mariela Herrera Checa; Andrea Leonor Rodríguez Ramos; Luis Alberto Barrera Delgado; María Fernanda Sánchez Pilozo

\section{Imagen $\mathbf{N}^{\circ} 2$ Bocio Multinodular}

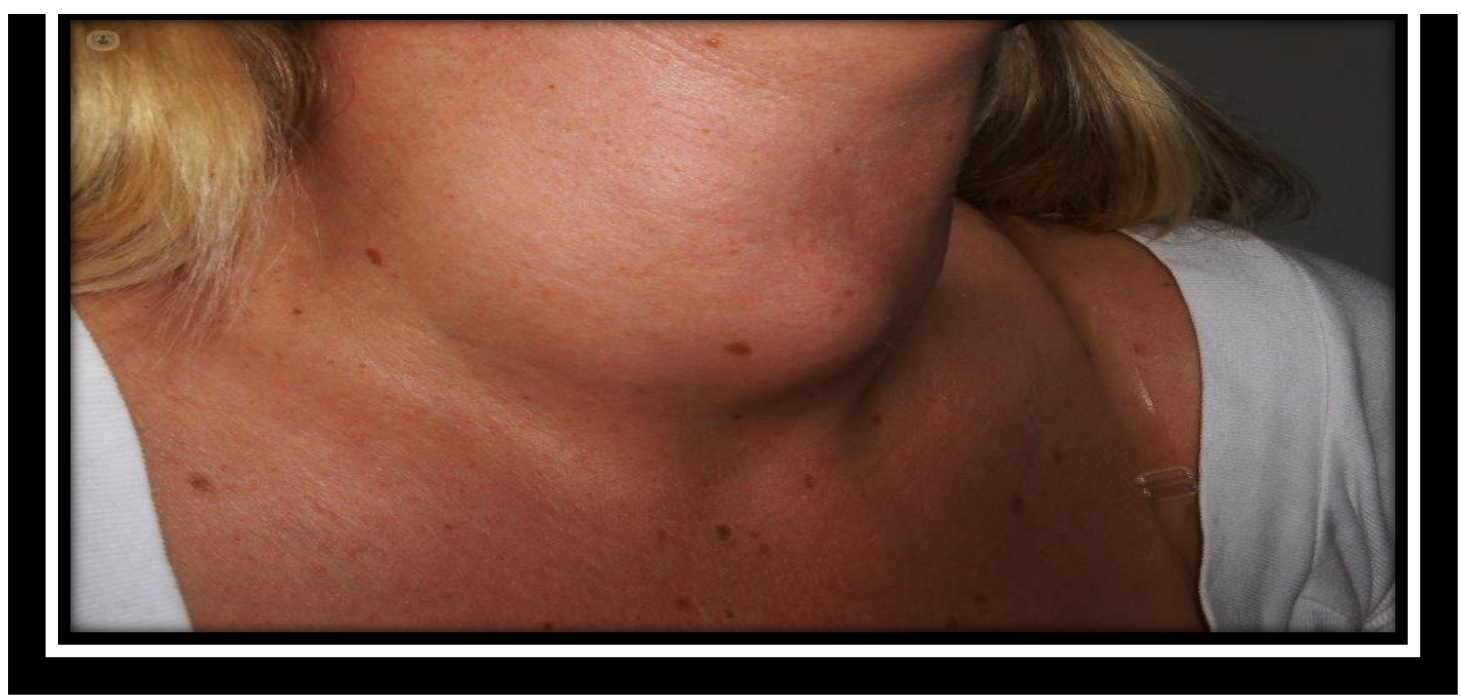

Fuente: Sánchez (ob.cit)

\section{Tratamiento Quirúrgico}

En general, en el bocio simple se recomienda seguir una actitud expectante con controles periódicos. En algunos casos muy seleccionados, por preferencias del paciente o cuando existen signos o síntomas de compresión, debe valorarse la indicación de la tiroidectomía subtotal bilateral. El tratamiento quirúrgico se indicará siempre en los pacientes con ENT y sospecha o diagnóstico de malignidad. Este grupo incluye a todos los enfermos con criterios clínicos de malignidad independientemente del diagnóstico citológico y también a todos los que presentan el diagnóstico citológico de proliferación folicular, aunque únicamente son malignos el 10-20\% de los NT con esta citología. La extensión de la cirugía (hemitiroidectomía, tiroidectomía subtotal o total) dependerá del diagnóstico definitivo de la lesión. (Revista Cubana de Endocrinología, 2004) 


\section{Bocio multinodular tratamiento quirúrgico}

Vol. 3, núm. 4., (2019)

Olga Mariela Herrera Checa; Andrea Leonor Rodríguez Ramos; Luis Alberto Barrera Delgado; María Fernanda Sánchez Pilozo

Los pacientes con adenoma tóxico o bocio multinodular tóxico son tributarios de tratamiento con fármacos antitiroideos $\mathrm{y}$, una vez conseguida la normo función, se planteará el tratamiento quirúrgico (hemitiroidectomía o tiroidectomía subtotal) o la administración de radio yodo. En general, la cirugía se indica en los enfermos más jóvenes y con NT de mayor tamaño. El radio yodo controla el hipertiroidismo y también puede disminuir el tamaño de los nódulos; aunque clásicamente se había reservado para los pacientes con riesgo quirúrgico importante, en el momento actual se utiliza de forma más amplia y con buenos resultados iniciales.

En los últimos años, se ha introducido la inyección local de etanol o de otras sustancias esclerosantes, guiada o no por ecografía, en el tratamiento de los adenomas tóxicos, con buenos resultados, en pacientes seguidos durante cortos períodos de tiempo. Esta técnica puede ser una alternativa al radio yodo y también se ha utilizado en quistes y en nódulos mixtos, después de su vaciamiento mediante PAAF, con el objetivo de prevenir la recidiva. Los pacientes con ENT e hipotiroidismo se tratarán con dosis sustitutivas de levotiroxina sódica, siempre que no exista sospecha de enfermedad maligna, en cuyo caso se indicará la intervención quirúrgica.

Ante un paciente con ENT, citología benigna y normo función tiroidea, existen diferentes opciones de tratamiento. La cirugía es una de ellas, pero en más del 90\% de los casos resulta innecesaria. Otra alternativa terapéutica, introducida recientemente en algunos de estos pacientes y con la que se obtienen resultados aceptables, es la administración de radio yodo. La utilidad del tratamiento de los pacientes portadores de una ENT benigna con levotiroxina sódica dosis que reducen la producción de TSH es aún un tema de debate. Su hipotética efectividad se basa en la teoría clásica de la influencia de la TSH en el crecimiento del tejido tiroideo, aunque estudios más recientes demuestran la importancia de otros factores diferentes de la TSH en el desarrollo 


\section{Bocio multinodular tratamiento quirúrgico}

Vol. 3, núm. 4., (2019)

Olga Mariela Herrera Checa; Andrea Leonor Rodríguez Ramos; Luis Alberto Barrera Delgado; María Fernanda Sánchez Pilozo

de los NT, algunos autores continúan utilizando este tratamiento. Así, en los últimos 20 años, se han publicado diferentes trabajos que evalúan la eficacia de la administración de dosis variables de levotiroxinapara conseguir disminuir el tamaño o inducir la desaparición de los NT con resultados contradictorios. (Navarro Despaigne, 2015)

Al analizar la relación riesgo/beneficio de este tratamiento no deben olvidarse sus posibles efectos secundarios tanto sobre el metabolismo óseo como sobre la morbilidad cardiovascular. Por ello, en las recomendaciones más recientes sobre el tratamiento que reduce la producción de TSH en la ENT, se consideran de forma separada, por su diferente riesgo, la población masculina y femenina y, dentro de ésta, las mujeres pre menopáusicas de las menopáusicas. En general, se aconseja una actitud más conservadora en los grupos de mayor riesgo y administración de la dosis mínima posible de levotiroxina y durante menos tiempo en estos grupos de pacientes. Sin embargo, en espera de estudios más amplios y con más tiempo de seguimiento, la mayoría de autores recomienda mantener una conducta expectante con revaloraciones periódicas en los pacientes con ENT benigna. El tratamiento con levotiroxina sódica está contraindicado si la concentración de TSH es inferior a $0,1 \mathrm{mU} / 1$ por el riesgo de desarrollar hipertiroidismo.

Según (Fernández Morocho, 2019), la tiroidectomía se encuentra descrita como el procedimiento endocrino más comúnmente practicado por cirujanos generales, siendo además la cirugía cervical más frecuente. En el caso de los pacientes geriátricos presentan, respecto al grupo control, mayor tiempo de evolución del bocio (160 frente a 87 meses; $p=0,0321$ ), mayor presencia de sintomatología (el 60 frente al 41\%; p = 0,0001) y mayor porcentaje de componente intratorácico (el 63 frente al 37\%; p =0,0001). Respecto a las indicaciones de la cirugía destaca 


\section{Bocio multinodular tratamiento quirúrgico}

Vol. 3, núm. 4., (2019)

Olga Mariela Herrera Checa; Andrea Leonor Rodríguez Ramos; Luis Alberto Barrera Delgado; María Fernanda Sánchez Pilozo

una mayor indicación por clínica compresiva (el 43 frente al 21\%; $\mathrm{p}=0,0012$ ). Se presentaron complicaciones postoperatorias en el $40 \%$ de los pacientes $(n=32)$, índice superior al del grupo control (el 28\%; $\mathrm{p}=0,0113$ ), aunque en un alto porcentaje fueron complicaciones transitorias. Como complicaciones definitivas se presentaron 2 lesiones recurrenciales (2,5\%). En todos los casos la sintomatología preoperatoria remitió, y sólo se asoció un carcinoma tiroideo en 3 pacientes $(3,7 \%)$, uno de ellos anaplásico.

En consecuencia, el bocio multinodular intervenido en pacientes añosos presenta una mayor evolución y un mayor componente intratorácico, por lo que la indicación de cirugía se produce de forma restrictiva. Con un buen control de las comorbilidades y una intervención programada los resultados en cuanto a la morbimortalidad son semejantes a los obtenidos en edades más tempranas. Por lo tanto, la eliminación de toda la glándula tiroides o de parte de ella (tiroidectomía total o parcial) es una opción si tienes un bocio grande que sea incómodo o cause dificultad al respirar o tragar o, en algunos casos, si tienes un bocio nodular que causa hipertiroidismo. La cirugía se reserva para pacientes con bocio de gran tamaño (> 80 gr o grado III), con síntomas compresivos así como con citología sospechosa de malignidad. Según la ATA y LATS, la recomendación en cuanto a la técnica quirúrgica es la tiroidectomía casi total o total sobre todo para aquellos pacientes con sospecha de malignidad

Como resultado de la utilización del tratamiento quirúrgico del bocio multinodular, agrega en el paciente bienestar, Pues, se hace una reducción drástica de la tumoración, descomprensión inmediata de las estructuras afectada, mejoría inmediata de los síntomas, diagnóstico histológico definitivo. Aunque existen diferentes riesgos entre ellos: anestésico, edad y comorbilidad. Por ello, el seguimiento postquirúrgico inmediato se basa en la vigilancia de los 


\section{Bocio multinodular tratamiento quirúrgico}

Vol. 3, núm. 4., (2019)

Olga Mariela Herrera Checa; Andrea Leonor Rodríguez Ramos; Luis Alberto Barrera Delgado; María Fernanda Sánchez Pilozo

niveles de calcio sérico, lo cual debe hacerse en las siguiente 6-12 horas posterior a la cirugía. En caso de hipocalcemia transitoria debe implementarse tratamiento con gluconato de calcio vía intravenosa y posteriormente carbonato de calcio por vía oral (dosis: 1500- $2000 \mathrm{mg} /$ día) sólo o en combinación con calcitriol (dosis: 0,5-1 $\mu \mathrm{g} /$ día) por 1-2 semanas.

\section{Conclusiones.}

De acuerdo con los planteamientos citados a lo largo de la descripción de los diversos eventos que conforman al presente artículo, hacen posible reflexionar en cuanto a su importancia en el campo de las ciencias médicas como una respuesta favorable para la incursión en nuevas ideas aportantes en el tema seleccionado, entre las cuales se consideran las siguientes:

La tiroides es una glándula de importancia para el desarrollo de los seres humanos, su intervención en el crecimiento se hace significativa, además facilita la regulación de las hormonas que permiten controlar el calor y frío, mejoran las condiciones generales del ritmo cardíaco entre otros. Pero al mismo tiempo, cuando se presentan problemas de aumento o disminución de la producción de yodo, hace posible el surgimiento de diferentes enfermedades que ameritan ser diagnosticadas para agregar de forma inmediata el tratamiento correspondiente.

Es importante acotar que en las ciencias médicas los endocrinólogos como especialistas encargados del estudio de la tiroides, han encontrado como resultado de las actividades investigativas, diferentes enfermedades tales como: hipertiroidismo, hipotiroidismo, bocio nodulares donde se identifica el multinodular y el cáncer de tiroides. Al hacer referencia al bocio multinodular, se puede indicar que el mismo se evidencia con un abultamiento de la glándula, que puede ser interno y externo, su presencia hace posible que los pacientes diagnosticados con 


\section{Bocio multinodular tratamiento quirúrgico}

Vol. 3, núm. 4., (2019)

Olga Mariela Herrera Checa; Andrea Leonor Rodríguez Ramos; Luis Alberto Barrera Delgado; María Fernanda Sánchez Pilozo

la respectiva enfermedad, puede iniciar tratamiento con levotiroxina; pero dependiendo de su actuación los cirujanos toman la decisión de aplicar cirugía como tratamiento final.

De allí, que la utilización del tratamiento quirúrgico en el bocio multinodular simple varía en función de la extensión de la enfermedad y de las preferencias del cirujano. La enfermedad puede aparecer inicialmente como un nódulo solitario, pero con el tiempo puede generalizarse, además hace que el paciente logre cambios efectivos en su vida, al agregar de forma inmediata la eliminación de los síntomas comprensivos, sin embargo, después de la tiroidectomía las complicaciones más importantes es la parálisis del nervio recurrente debido a que este nervio puede ser lesionado durante la cirugía.

De igual manera se puede producir un amplio espectro de complicaciones en la voz, mecanismos de la deglución o ambos, cambio temporal o permanente en la voz. Si el bocio reaparece (recurrencia del bocio) algún tiempo después de la tiroidectomía, puede ser necesaria otra intervención quirúrgica. La cual, es más complicada que la inicial debido al tejido cicatrizal que dificulta la identificación de los nervios u otros tejidos importantes. También existe la posibilidad de que la tiroidectomía subtotal, que se considera algo más segura que la tiroidectomía total, pueda dejar cáncer tiroideo sin detectar en el sitio.

\section{Bibliografía.}

Copello Millares, M., Merino Rodríguez, R., \& Carralero Zaldívar, M. (2016). Enfermedades relacionadas con la glándula tiroides. Correo Científico Médico, 20(4), 810-815.

Fernández Morocho, J. (2019). Validación de la punción aspiración con aguja fina guiada por ecografía en el diagnóstico de cáncer de tiroides. Hospital José Carrasco Arteaga, Cuenca, Ecuador, 2017. Cuenca, Ecuador: Universidad de Cuenca. 


\section{Bocio multinodular tratamiento quirúrgico}

Vol. 3, núm. 4., (2019)

Olga Mariela Herrera Checa; Andrea Leonor Rodríguez Ramos; Luis Alberto Barrera Delgado;

María Fernanda Sánchez Pilozo

Martin, M. (2016). Structure and function of the thyroid gland. Revista ORL, 7(2), 7-16.

Navarro Despaigne, D. (2015). Hormonas tiroideas en afecciones benignas y malignas del tiroides, de la certidumbre a la duda. Revista Cubana de Endocrinología, 26(3), 12-22.

Pernalete, S. (2016). Método de Investigación. Madrid: MORATA.

Revista Cubana de Endocrinología. (2004). Consenso en el diagnóstico y tratamiento de las afecciones del tiroides. Rev Cubana Endocrinol, 15(1), 1-2.

Rodríguez Ramos, J., Boffill Corrales, A., \& Rodríguez Soria, L. (2016). Factores de riesgo de las enfermedades tiroideas. Hospital del Seguro Social Ambato. Revista de Ciencias Médicas de Pinar del Río, 20(5), 628-638.

Sosa Martín, G., \& Ernand Rizo, S. (2016). Complicaciones derivadas de la tiroidectomía en el Hospital General "Calixto García". Revista Cubana de Cirugía, 55(4), 271-278.

$$
\text { (9) }(1)(9)
$$

RECONOCIMIENTO-NOCOMERCIAL-COMPARTIRIGUAL

CC BY-NC-SA

ESTA LICENCIA PERMITE A OTROS ENTREMEZCLAR, AJUSTAR Y CONSTRUIR A PARTIR DE SU OBRA CON FINES NO COMERCIALES, SIEMPRE Y CUANDO LE RECONOZCAN LA AUTORÍA Y SUS NUEVAS CREACIONES ESTÉN BAJO UNA LICENCIA CON LOS MISMOS TÉRMINOS. 